\title{
Extracting Visual Knowledge from the Web with Multimodal Learning
}

\author{
Dihong Gong, Daisy Zhe Wang \\ Department of Computer and Information Science and Engineering \\ University of Florida \\ \{gongd, daisyw\}@ufl.edu
}

\begin{abstract}
We consider the problem of automatically extracting visual objects from web images. Despite the extraordinary advancement in deep learning, visual object detection remains a challenging task. To overcome the deficiency of pure visual techniques, we propose to make use of meta text surrounding images on the Web for enhanced detection accuracy. In this paper we present a multimodal learning algorithm to integrate text information into visual knowledge extraction. To demonstrate the effectiveness of our approach, we developed a system that takes raw webpages and a small set of training images from ImageNet as inputs, and automatically extracts visual knowledge (e.g. object bounding boxes) from tens of millions of images crawled from the Web. Experimental results based on 46 object categories show that the extraction precision is improved significantly from $73 \%$ (with state-ofthe-art deep learning programs) to $81 \%$, which is equivalent to a $31 \%$ reduction in error rates.
\end{abstract}

\section{Introduction}

Recent progresses on computer vision research community such as large scale object detection [Girshick, 2015][Simonyan and Zisserman, 2014], age invariant face recognition [Gong et al., 2013; 2015], and region-to-phrase correspondences [Plummer et al., 2015], largely benefit from ever increasing amount of visual knowledge as training data. Collecting visual knowledge in a crowdsourcing manner, such as the ImageNet database [Deng et al., 2009] and Visipedia [Perona, 2010], has major limitation in the lack of both diversity and scalability. In addition, manually annotating large collection of images is usually an expensive and time consuming process. For example, based on the most recent statistics, there are only $7.28 \%$ images annotated in the ImageNet database. Given these limitations, in this paper we explore an alternative approach to build large visual knowledge base by extracting visual knowledge from Web data.

Mining useful visual knowledge automatically from the Web can be challenging. Even with the recent state-of-theart visual object detection algorithms, the precision of content based image retrieval is still unacceptable. For example,
GoogLeNet [Szegedy et al., 2015] which finished at the $1^{\text {st }}$ place in the ImageNet Large-Scale Visual Recognition Challenge 2014, only achieves mean average precision of $43.9 \%$.

Given the limitation of visual knowledge extraction methods purely relying on visual content, this paper explores an alternative method to integrate multimodal information for better extraction accuracy. The Web is mostly a vast collection of unstructured information of various modalities (e.g. text, image, and video), where multimodal information is usually correlated. For instance, images of a news article usually illustrate the corresponding text content and meta text like alt or src of an image describes the content of that image. These observations suggest that a potentially greater visual knowledge extraction accuracy can be achieved by making use of information from alternative modalities.

\section{Related Work and Our Contributions}

Traditionally, visual knowledge bases such as ImageNet [Deng et al., 2009] and Visipedia [Perona, 2010] are constructed by manual annotations with motivated teams of people or power of crowds. These approaches however are quite limited because annotations become expensive, prone to errors and do not scale. To overcome these disadvantages, recent studies have been focused on leveraging machine learning technologies to reduce human intervention. For example, Vijayanarasimhan et al. [Vijayanarasimhan and Grauman, 2014] proposed an active learning algorithm based on crowdsourcing using Amazon Mechanical Turk to train object detectors with crawled data. Later, Chen et al. [Chen $e t$ al., 2013b] presented a completely autonomous system called Never Ending Image Learner (NEIL) to automatically mine visual knowledge from the Web. While these systems are designed to reduce the human intervention for visual knowledge mining, their retrieval precision is still quite low (e.g. NEIL has mean average precision of $51 \%$ for 15 object categories) due to limited source of information.

There have been an increasing research interest for multimodal learning in the recent years. The general goal of multimodal learning is to utilize information across multiple modalities (e.g. text, image, video, and audio) for either enabling cross-modality query or improving retrieval accuracy for a wide variety of machine learning tasks. For instances, Zhu et al. [Zhu et al., 2015] proposed a scalable algorithm to build multimodal knowledge base for an- 
swering visual queries. Their system takes annotated multimodal data (e.g. images with text descriptions and attributes) as input, and establishes relations between entities using MRF models. The system however doesn't completely solve the automatic visual knowledge mining problem because it relies on annotated multimodal data, the acquisition of which is another challenging problem yet to be addressed. Other representative approaches such as [Kiros et al., 2014; Norouzi et al., 2013; Lazaridou et al., 2015; Frome et al., 2013] primarily focus on learning semantic embeddings using deep neural networks for multimodal object representations. The basic idea of these approaches is to map objects in different modalities into a common vector space so that correspondence between multimodal objects can be established. For example, Kiros et al. [Kiros et al., 2014] proposed a multimodal skip-gram model to learn word embeddings closely related to the corresponding vision concepts (e.g. embedding of word kitten is close to embedding of visual objects in cat category). Similarly, Frome et al. [Frome et al., 2013] developed a system called "DeViSE" that learns to transform embeddings from visual modality to textual modality to allow prediction of unseen visual categories based on text labels (so called Zero-Shot Learning [Norouzi et al., 2013]). However, like the system by Zhu et al. [Zhu et al., 2015], all of these methods rely on image data manually annotated (e.g. annotation is noise-free) with text descriptions by human workers (e.g. Flickr 8K [Hodosh et al., 2013] and ImageNet [Deng et al., 2009]). Annotating images with text descriptions is another challenging problem yet to be solved. Consequently, these approaches may not be suitable for opendomain visual knowledge extraction, where noise-free image descriptions are usually unavailable.

Our work is closely related to the aforementioned approaches, with major contributions summarized as follows:

- The major novelty of this paper is to present a multimodal learning approach to integrate meta text surrounding web images for large scale open-domain visual knowledge extraction. Compared to existing approaches, our approach is proved to be effective for realworld web data that is noisy, incomplete or redundant.

- We develop a sophisticated end-to-end system for largescale visual knowledge extraction from real web data. Unlike existing multimodal approaches which test on standard datasets, in this paper we take hundreds of millions of raw webpages as input, and automatically extract visual object bounding boxes as outputs for testing.

- Finally, we demonstrate a significant improvement in extraction precision over the state-of-the-art visual object detection algorithms. Experimental results based on 46 object categories show that by making use of textual and visual information jointly, the extraction precision is improved significantly from $73 \%$ to $81 \%$, which is equivalent to a $31 \%$ reduction in error rates.

\section{Multimodal Embeddings}

In this section, we describe an algorithm to learn embeddings for textual words and visual concepts. The learned represen- tative embeddings in continuous space preserve relative distance between multimodal objects, such that if two objects have similar meaning (e.g. "car" and "truck"), their embeddings are also close to each other.

\subsection{Review of Skip-Gram Model}

Our algorithm is closely related to the skip-gram model [Guthrie et al., 2006] which is a language modeling algorithm used to learn embeddings of text words. Given text corpus, the training objective of the Skip-Gram model is to find word representations that are useful for predicting the surrounding words in a sentence. Mathematically, it maximizes the objective function

$$
\frac{1}{T} \sum_{t=1}^{T} \sum_{-c \leq j \leq c, j \neq 0} \log p\left(w_{t+j} \mid w_{t}\right),
$$

where $w_{1}, w_{2}, \ldots w_{T}$ is a sequence of training words in the corpus, and $c$ is the size of the window around target $w_{t}$. In the basic Skip-Gram model, the conditional probability $p\left(w_{t+j} \mid w_{t}\right)$ is defined using the softmax function as

$$
p\left(w_{O} \mid w_{I}\right)=\frac{\exp \left(v_{w_{O}}^{\prime}{ }^{T} v_{w_{I}}\right)}{\sum_{w=1}^{W} \exp \left(v_{w}^{\prime}{ }^{T} v_{w_{I}}\right)},
$$

where $v_{w}$ and $v_{w}^{\prime}$ are the "input" and "output" vector representations of $w$, and $W$ is the size of the vocabulary. Due to the normalization term in denominator, the Equation (2) requires $O(W)$ time complexity, which makes it computionally impractical since the size of vocabulary is usually very large (e.g. $10^{5}-10^{7}$ ). To speedup the computation, hierarchical softmax [Mikolov et al., 2013] is proposed to approximate the standard softmax in Equation (2) with a binary tree, which reduces the time complexity from $O(W)$ to $O(\log (W))$ on average. In this paper, we shall apply the hierarchical softmax whose implementation is based on Google word2 $\mathrm{vec}^{1}$.

\subsection{Definition}

Suppose we have a set of $N$ images from which visual knowledge is to be extracted, denoted as

$$
\mathcal{I}=\left\{\left(I_{n}, T_{n}\right) \mid n=1 \ldots N\right\},
$$

where $I_{n}$ represents an image, and $T_{n}$ denotes a set of text phrases describing image $I_{n}$. Then we apply visual object detection program on each of the image $I_{n}$, and arrive at a set of detected visual objects denoted as

$$
\mathcal{I}_{\mathcal{D}}=\left\{\left(D\left(I_{n}\right), T_{n}\right) \mid I_{n} \in \mathcal{I} \wedge \operatorname{card}\left(D\left(I_{n}\right)\right)>0\right\},
$$

where $D(\cdot)$ is a detection operator gives a set of detected visual categories, and card $(\cdot)$ is the cardinality of a set. We only retain images that have at least one detected object from predefined set of visual categories (denoted as $\mathcal{C}$ ).

We have two vocabularies, one for text and one for image. The text vocabulary (denoted as $\mathcal{V}$ ) is derived from text phrases associated with images in $\mathcal{I}_{\mathcal{D}}$, and visual vocabulary (denoted as $\mathcal{C}$ ) is derived from visual categories.

\footnotetext{
${ }^{1}$ https://code.google.com/archive/p/word2vec
} 


\subsection{Formulation}

Our goal is to learn embeddings for text phrase $x \in \mathcal{V}$ and visual category $y \in \mathcal{C}$, such that:

- The embeddings $\vec{v}(x)$ and $\vec{v}(y)$ are close to each other (e.g. in Euclidean $n$-space with cosine distance) if text phrase $x$ is an appropriate description of image category $y$. For example, $\vec{v}($ kitten $)$ should be close to $\vec{v}(\mathbf{c a t})$, where kitten $\in \mathcal{V}$ and cat $\in \mathcal{C}$, since kitten is an appropriate description of cat.

- The embeddings $\vec{v}\left(y_{1}\right)$ and $\vec{v}\left(y_{2}\right)$ are close to each other if objects in image category $y_{1}$ co-occur with objects in image category $y_{2}$ with high frequency. Two objects cooccur if they occur in the same image. For example, $\vec{v}$ (car wheel) is close to $\vec{v}(\mathbf{c a r})$ because objects of car wheel and car co-occur with high frequency.

These two items are essential. The first item identifies a set of important text phrases that are useful for image retrieval. For example, if we have text kitten as tag of an image, then the confidence about that image contains objects in cat category becomes higher. The second item identifies important visual categories that are useful for image retrieval of other visual categories. For example, if we have detected an object of car, then the confidence of that image contains car wheel objects becomes higher. This kind of visual co-occurrence regularity has been shown to be useful in improving detection precision in NEIL [Chen et al., 2013a].

Our model jointly encodes the intuition of multimodal information and visual co-occurrence regularity, by maximizing the following objective function:

$$
\frac{1}{\operatorname{card}\left(\mathcal{I}_{\mathcal{D}}\right)} \sum_{(D, T) \in \mathcal{I}_{\mathcal{D}}} \sum_{\substack{u \neq v \\ u, v \in D \cup T}} \log p(v \mid u)
$$

For each image, we have a set of visual categories $D$ obtained from image detection (e.g. if we detect car and car wheel objects in an image, then $D=\{$ car, car wheel $\}$ ), and a set of text phrases $T$ obtained from extracting text information surrounding the image (e.g. if title of an image contains text phrases autmobile and dealer then $T=$ \{autmobile, dealer $\}$ ). For an image we calculate the sum of conditional probabilities predicting elements between each other, and the overall objective function maximizes average of these sums. It can be verified that this objective function satisfies the two items we have previously declared.

We observe that the multimodal objective function in Equation (4) can be transformed into objective function of the Skip-Gram model in Equation (1) by:

1. Mapping each object $u_{n} \in D\left(I_{n}\right) \cup T_{n}$ to a word $w_{t}$;

2. Mapping a set of multimodal objects $D\left(I_{n}\right) \cup T_{n}$ to words $w_{t-c} \ldots w_{t+c}$ around the target word $w_{t}$;

Thus, with these transformations we can solve the optimization problem in Equation (4) using the standard Skip-Gram model. In this paper, we have applied the hierarchical softmax version of the Skip-Gram model. After embeddings are learned, we normalize the embedding vectors by dividing their $L_{2}$ norms such that all vectors are of unit magnititude.

\subsection{Image Tagging}

The image tagging program automatically assigns each image a set of noun phrases (tags) that best describe the image [Chen et al., 2013a]. We extract tags of each image based on both image meta and web page context information, following these steps:

- Retrieve top-k noun phrases (denoted as TopNP) from a web page that containing the target image to be tagged. The importance of noun phrases are measured by tfidf score

$$
t f i d f(t, d)=0.5+0.5 \frac{f_{t, d}}{\max \left\{f_{t^{\prime}, d}: t^{\prime} \in d\right\}} \times \log \frac{N}{n_{t}},
$$

where $d$ represents the webpage document, $f_{t, d}$ is the frequency of term $t$ in document $d, N$ is the total number of webpages, and $n_{t}$ is the total number of webpages containing the term $t$. We found $k=30$ performs well in our system.

- For each noun phrase in TopNP, if either src or alt attribute of $\langle\mathrm{img}\rangle$ tag contains the noun phrase, then assign it as a tag of the image.

- Retain top-k tags for each image with the highest tfidf scores. We set $k=3$ in our system.

The Table 1 illustrates some example images annotated with tags. We can see the extracted tags can be inaccurate or incomplete, which makes it challenging to apply the multimodal learning to predict visual objects. In the next section, we shall describe an algorithm using structure learning approach to efficiently select an optimal set of tags that are most useful for prediction.

\section{Structure Learning and Prediction}

In this section, we present a prediction model based on multimodal embeddings for visual knowledge extraction.

\subsection{Multimodal Vocabulary}

The multimodal vocabulary, denoted as $\mathcal{W}$, is a union of text vocabulary $\mathcal{V}$ and visual vocabulary $\mathcal{C}$.

$$
\mathcal{W}=\mathcal{V} \cup \mathcal{C}
$$

In our system, we require a word to have minimum frequency of 5 to be considered as a member of $\mathcal{W}$. Then, an image in Equation (3) can be represented using the $\mathcal{W}$ as

$$
\mathcal{I}_{\mathcal{D}}=\left\{W_{n} \mid W_{n}=\left(D\left(I_{n}\right) \cup T_{n}\right) \cap \mathcal{W}\right\},
$$

where $W_{n}$ is a set of words from vocabulary $\mathcal{W}$ corresponding to image $I_{n}$. For a concrete example, let's suppose we have an image $I_{n}$, where

$$
D\left(I_{n}\right)=\{\text { car, car wheel }\}
$$

indicates that the visual detector can detect objects in categories of car, car wheel from $I_{n}$, and

$$
T_{n}=\{\text { automobile, dealer }\}
$$

means $I_{n}$ has two text tags: "automobile" and "dealer". Then, according to the definition,

$$
W_{n}=\{\text { car, car wheel, automobile, dealer }\} .
$$

In the next section, we shall describe an effective learning algorithm to predict if objects of a category (e.g. car) present in $I_{n}$ using $W_{n}$. 


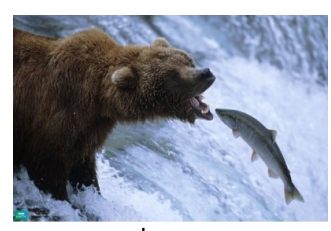

bear

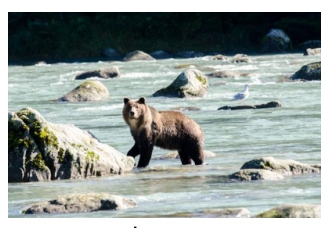

tours

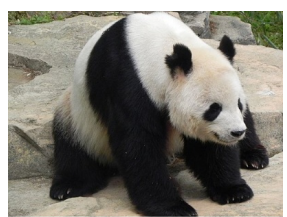

national zoo, zoo

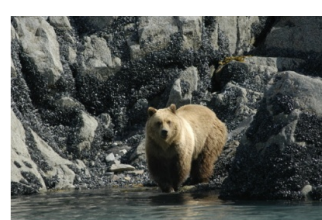

bears, glacier bay, lewis

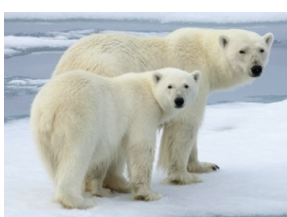

bears

Table 1: Example web images with tags automatically annotated

\subsection{Structure Learning}

We predict the visual objects in an image based on the multimodal words $W_{n}$ describing the image. Mathematically, we model the probability that an image $I_{n}$ contains objects of category $c$ with a logistic regression model

$$
p_{\boldsymbol{\theta}}\left(c \mid W_{n}\right)=\frac{e^{\theta_{0}+\sum_{w_{k} \in W_{n}, w_{k} \neq c} \theta_{k} \vec{v}\left(w_{k}\right)^{T} \vec{v}(c)}}{1+e^{\theta_{0}+\sum_{w_{k} \in W_{n}, w_{k} \neq c} \theta_{k} \vec{v}\left(w_{k}\right)^{T} \vec{v}(c)}},
$$

where $W_{n}$ is a set of multimodal words describing image $I_{n}$, and $\theta_{0}, \theta_{1}, \ldots$ (collectively denoted as $\boldsymbol{\theta}$ ) are the bias and combination coefficients. When summarizing over $W_{n}$, we exclude the word $c$ because $\vec{v}\left(w_{k}\right)^{T} \vec{v}(c)=1$ is constant when $w_{k}=c$ (all vectors are $L_{2}$ normalized). Note that each multimodal word is corresponding to one $\theta$ (excluding $w_{k}=c$ ), so the total number of parameters is $\operatorname{card}(\mathcal{W})$, including bias $\theta_{0}$. The operator $\vec{v}(\cdot)$ converts a word into its corresponding distributed vector representation as before. In this manner, the probability is determined by weighted combination of $W_{n}$. Note that we train one prediction model per category, thus different categories have different $\boldsymbol{\theta}$ parameters.

To learn the model parameters $\boldsymbol{\theta}$ w.r.t. category $c$, we maximize the following regularized objective function

$L(\boldsymbol{\theta}, c)=\sum_{n \in \mathcal{P}} \frac{\ln p_{\boldsymbol{\theta}}\left(c \mid W_{n}\right)}{\operatorname{card}(\mathcal{P})}+\sum_{n \in \mathcal{N}} \frac{\ln \left(1-p_{\boldsymbol{\theta}}\left(c \mid W_{n}\right)\right)}{\operatorname{card}(\mathcal{N})}-\lambda|\boldsymbol{\theta}|_{1}$,

where $\mathcal{P}$ denotes a set of indices for image samples in $\mathcal{I}_{\mathcal{D}}$ that are relevant to category $c$, and $\mathcal{N}$ denotes the irrelevant samples. An image is relevant to category $c$ if visual detector detects objects of category $c$ (e.g. $c \in W_{n}$ ). Equation (7) is a balanced and regularized version of the log likelihood. Usually the number of negative training samples is much more than the positive samples, so we balance uneven number of training samples by dividing the cardinalities. The $L_{1}$ regularization term is used to encourage sparse solutions while at the same time keep the optimization problem convex. We need sparse solution for $\boldsymbol{\theta}$ because the size of $\mathcal{W}$ is usually very large (e.g. $10^{5}-10^{7}$ ) and only a very small fraction of words are useful for specific category $c$. With proper sparsity level, words that are not useful will have zero $\theta$ value, which makes these useless words inactive at prediction stage. Intuitively speaking, in the example of Equation (5), we expect word "image" to have zero $\theta$ value when $c$ is car because "image" is not closely related to category car. On the contrary, if $\theta$ value of "image" is nonzero, then this word can easily lead to false positive prediction if $\theta$ is positive and false negative detection if $\theta$ is negative.

The prediction model in Equation (6) predicts visual objects based on both visual co-detection and text information.
For example, assuming that target category $c$ is car and $W_{n}$ is given by Equation (5), then Equation (6) predicts that image $I_{n}$ contains car objects based on weighted combination of: car wheel, automobile, image. Intuitively, the confidence about $I_{n}$ containing car objects becomes higher if we know that car wheel objects can be detected from $I_{n}$, which we called visual co-detection information. Similarly, the same confidence increases as we learn that text tags of $I_{n}$ contains "automobile", which we called text information.

\subsection{Unifying Predictions}

The probabilistic model in Equation (6) exploits both visual co-detection and text information. However, this probabilistic function doesn't take the prediction given by object detectors into consideration. To include the confidence predicted by object detectors, denoted as $q\left(c \mid I_{n}\right)$, we unify the two predictions to give the final scoring function as

$$
\operatorname{score}\left(I_{n}, c\right)=p_{\boldsymbol{\theta}}\left(c \mid W_{n}\right) \cdot q\left(c \mid I_{n}\right),
$$

where $W_{n}$ is a set of multimodal words corresponding to image $I_{n}$ as before. As a result, for each visual category $c$ we rank all candidate images in $\mathcal{I}_{\mathcal{D}}$ by this confidence score and then retrieve the top candidates as output.

\section{Experiments and Results}

In this section, we evaluate the proposed multimodal algorithm in the context of Web visual knowledge mining.

\subsection{Dataset}

We evaluate our approach based on a collection of web pages and images derived from the Common Crawl dataset [Smith et al., 2013] that is publicly available on Amazon S3. The entire Common Crawl dataset comprises billions of raw webpages in warc compressed format, and for our study we take a subset of the data with hundreds of millions of webpages. These webpages are processed following these steps:

1. Parse the HTML webpages, with a $\mathrm{C}++$ open-source program Gumbo-Parser by Google ${ }^{2}$.

2. Extract all image urls of each web page, along with alt and src attributes. We only retain images whose dimension (shorest edge) is at least 150 pixels.

3. Clean meta and spam from web pages to obtain plain text, then tokenize and apply part-of-speech tagging. The part of speech tagger is based on Tree-Tagger program [Schmid, 1995] for best computational efficiency.

\footnotetext{
${ }^{2}$ https://github.com/google/gumbo-parser
} 
4. Extract nouns and noun phrases. The nouns are extracted based on part-of-speech tag of a word. The noun phrases are extracted based on the following rules:

- Common noun phrases (e.g. "computer monitor") consist of a sequence of consecutive common nouns;

- Proper noun phrases (e.g. "National Aeronautics and Space Administration") are a sequence of proper nouns optionally connected by conjunction or preposition.

5. Assign a set of tags to images by running the image tagging program described in Section 3.4. Then download images from the Web based on image urls in a distributed manner using Amazon S3 and EC2 (40 concurrent spot instances).

This results in a collection of around 10 million tagged images for our study.

\subsection{Experimental Settings}

In this section, we describe the detailed system parameters, baseline approach and experimental procedures.

\section{System parameters}

For each image we retain top 3 tags of the highest tfidf scores, and images are resized to fit a $217 \times 217$ bounding box. For multimodal embedding, we set the dimension of vector representations as 500 (we found that dimensions between 100 and 1000 give similar results) according to the recommendation from [Frome et al., 2013]. For structure learning, we tune the $\lambda$ parameter in Equation (7) on training data such that the number of non-zero elements is around 100 for the $\boldsymbol{\theta}$ parameter. We observed that the performance is stable when $\boldsymbol{\theta}$ has number of non-zeros elements between 50 and 200. In this paper, we consider 46 visual categories that are taken from the ImageNet [Deng et al., 2009] database. Each category is initialized with 250 seed images from the ImageNet with annotated object bounding boxes, which are then used to train visual object detector. For visual detection, we use the Fast R-CNN [Girshick, 2015] (a recent state-of-the-art deep learning algorithm) as our object detection program. The reasons we use Fast R-CNN are due to both its computational efficiency and being able to achieve accuracy that is comparable to other state-of-the-art approaches. The CaffeNet models with feature dimension of 4096 were trained on a NVIDIA Tesla K40c GPU. All detection parameters were set as default ${ }^{3}$. The trained detector was then applied to all images, which took around 35 days to complete the detection of 10 million images on a 16-core server machine with NVIDIA Tesla K40c GPU.

\section{Baseline approach}

The thesis explored in this paper is that a much greater extraction accuracy for Web visual knowledge extraction can be achieved by exploiting multimodal information. Thus, for each visual category $c$ our comparative baseline ranks all candidate images in $\mathcal{I}_{\mathcal{D}}$ merely based on visual information by

\begin{tabular}{llll}
\hline & & Precision $(\boldsymbol{\%})$ \\
Category & \#Det. & Uni. & Mul. \\
\hline beach & 11,457 & 74 & $91(+17)$ \\
bear & 9,751 & 72 & $90(+18)$ \\
bed & 92,024 & 95 & $100(+5)$ \\
bedroom & 3,318 & 96 & $100(+4)$ \\
bee & 4,477 & 59 & $70(+11)$ \\
boat & 10,215 & 74 & $90(+16)$ \\
car & 110,898 & 97 & $100(+3)$ \\
car mirror & 15,756 & 22 & $21(-1)$ \\
car tire & 10,180 & 33 & $48(+15)$ \\
car wheel & 75,554 & 87 & $100(+13)$ \\
cellular telephone & 69,768 & 93 & $100(+7)$ \\
chair & 81,642 & 92 & $100(+8)$ \\
civilian clothing & 182,826 & 69 & $73(+4)$ \\
computer keyboard & 14,959 & 23 & $31(+8)$ \\
crane & 4,262 & 31 & $45(+14)$ \\
female child & 402,921 & 93 & $100(+7)$ \\
fish & 20,694 & 9 & $29(+20)$ \\
game fish & 3,840 & 60 & $84(+24)$ \\
hand-held computer & 106,263 & 95 & $95(+0)$ \\
helicopter & 7,952 & 96 & $99(+3)$ \\
insect & 29,919 & 93 & $99(+6)$ \\
jersey & 134,964 & 100 & $100(+0)$ \\
kitchen & 88,884 & 82 & $89(+7)$ \\
laptop & 56,676 & 78 & $100(+22)$ \\
lifeboat & 6,671 & 63 & $71(+8)$ \\
locomotive & 6,643 & 83 & $90(+7)$ \\
man's clothing & 300,496 & 94 & $99(+5)$ \\
\hline microwave & 69,707 & 83 & $88(+5)$ \\
\hline musical instrument & 67,475 & 14 & $10(-4)$ \\
people & 468,914 & 98 & $100(2)$ \\
pizza & 225,795 & 72 & $67(-5)$ \\
racer & 78,487 & 94 & $100(+6)$ \\
riverbed & 19,689 & 78 & $82(+4)$ \\
salmon & 20,652 & 75 & $90(+15)$ \\
school bus & 6,964 & 54 & $49(-5)$ \\
seashore & 113,937 & 68 & $82(+14)$ \\
skirt & 117,309 & 88 & $98(+10)$ \\
sky & 161,540 & 95 & $100(+5)$ \\
suspension bridge & 5,841 & 30 & $48(+18)$ \\
table & 150,542 & 84 & $90(+6)$ \\
television & 45,690 & 19 & $45(+26)$ \\
truck & 24,263 & 92 & $97(+5)$ \\
vehicle & 5,446 & 88 & $97(+9)$ \\
wading bird & 4,371 & 91 & $98(+7)$ \\
warplane & 32,506 & 95 & $99(+4)$ \\
window & 275,872 & 75 & $92(+17)$ \\
\hline Average & $\mathbf{8 1 , 6 9 6}$ & $\mathbf{7 2 . 9 5}$ & $\mathbf{8 1 . 4 3 ( + 8 . 4 8 )}$ \\
\hline & & & \\
\hline
\end{tabular}

Table 2: The comparison of precision between Multimodal (Mul.) and Unimodal (Uni.) based on top 1,000 detections of each category (totally 46 categories). The \#Det. is total number of detected objects for a category from 10 million Web images (some images don't contain objects of interest).

\footnotetext{
${ }^{3}$ https://github.com/rbgirshick/fast-renn
} 


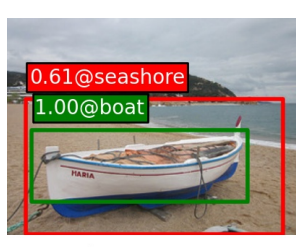

tossa, mar

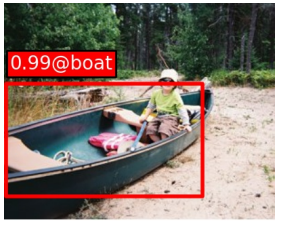

canoe

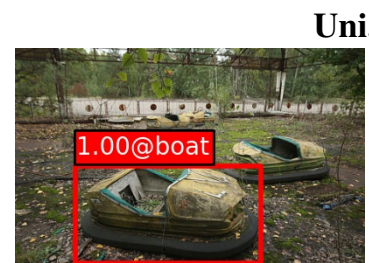

ukraine, chernobyl

.

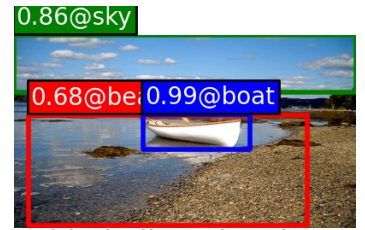

whitehall, maine, boat
Uni.@boat

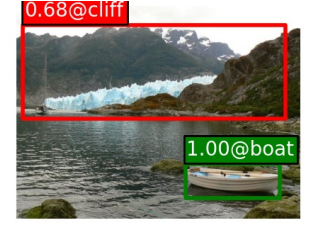

south america, travel, park

@boat

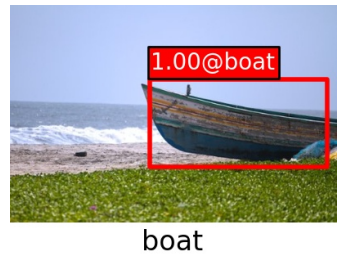

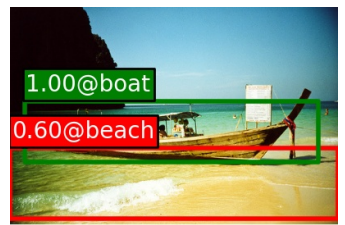

thailand

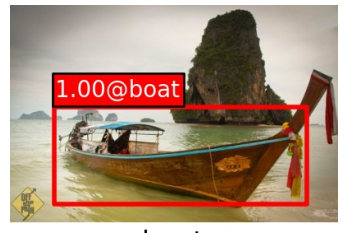

boat

Table 3: The comparison of top extracted objects for the unimodal (Uni.) and multimodal (Mul.) approaches based on three example visual categories. Each image is visualized with object bounding boxes along with the corresponding confidence scores given by the Fast-RCNN visual object detector. The text descriptions on the bottom of each image are extracted automatically by parsing web pages using our Image Tagging algorithm.

scoring function

$$
\operatorname{score}\left(I_{n}, c\right)=q\left(c \mid I_{n}\right),
$$

where $q\left(c \mid I_{n}\right)$ is confidence score predicted by object detector. We used the state-of-the-art visual object detection program (Fast R-CNN) as the baseline object detector.

\section{Experimental procedures}

We evaluate our approach by comparing the quality of the extracted visual knowledge. For each visual category, we rank all relevant images by scoring functions in Equation (8) and (9) respectively, and then retrieve the top- $k$ images with the highest scores as output. The precision of output images of category $c$ are estimated by

$$
\operatorname{Precision}(c, k)=\frac{\# \text { relevant }\left(S_{k}, c\right)}{\operatorname{card}\left(S_{k}\right)} .
$$

The $S_{k}$ is a set of images sampled randomly from top- $k$ retrieved images, and \#relevant $\left(S_{k}, c\right)$ denotes the number of images in $S_{k}$ that contains correct detection of objects in visual category $c$. In our paper, $k$ is set to be 1,000 and the size of $S_{k}$ is set to be 100 . Consequently, we compare the quality of the first 1,000 retrieved images based on estimation of 100 random samples from that 1,000 images. Retrieved images of different approaches are mixed together and then the correctness of individual retrieval is verified by human workers.

\subsection{Results}

\section{Quantative evaluation}

We first present our quantitive evaluation results by comparing the proposed multimodal algorithm (Mul.) against the baseline unimodal approach (Uni.). We estimated the precision of each visual category following the procedures described in Section 5.2, and results are shown in Table 2. In the table, the \#Det. column is the total number of detected visual objects of a category from 10 million image corpus based on
Fast R-CNN detector (may contain false detections). Based on the result in Table 2, we see that for most of the visual categories, the proposed multimodal approach outperforms the unimodal baseline by a clear margin, which confirms the effectiveness of the proposed approach. On average, the multimodal approach has improved the precision by $\mathbf{8 . 4 8 \%}$, which is equivalent to a $\mathbf{3 1 \%}$ reduction in error rates.

\section{Illustrative examples}

To intuitively examine the effectiveness, we visualize extracted examples as shown in Table 3. Due to the limited space, we only illustrate the top four examples of boat category. From these examples, we conclude that the baseline Uni. approach extracts objects with the highest visual detection score (1st row), while the proposed Mul. approach leverages both text and visual information (2nd row). We also observe that the text description for images retrieved with Mul. (2nd row) is more consistent with the visual objects in the images. The second image in the first row is a false positive extraction, which also shows the unreliability of algorithms relying on single source of information.

\section{Conclusion}

We demonstrate an effective multimodal learning algorithm for visual knowledge extraction from the Web. We have developed a mutimodal visual knowledge extraction system which takes raw web pages as input and extract visual objects leveraging both textual and visual information in a fully automatic manner. Evaluational experiments show that when compared with the state-of-the-art algorithms purely relying on visual content, our multimodal algorithm significantly improves the visual knowledge extraction precision.

\section{Acknowledgements}

This work is supported by DARPA under FA8750-12-2-03482 (DEFT/CUBISM), and NSF IIS Award \#1526753. 


\section{References}

[Chen et al., 2013a] Minmin Chen, Alice Zheng, and Kilian Weinberger. Fast image tagging. In Proceedings of the 30th international conference on Machine Learning, pages 1274-1282, 2013.

[Chen et al., 2013b] Xinlei Chen, Abhinav Shrivastava, and Abhinav Gupta. Neil: Extracting visual knowledge from web data. In Proceedings of the IEEE International Conference on Computer Vision, pages 1409-1416, 2013.

[Deng et al., 2009] J. Deng, W. Dong, R. Socher, L.-J. Li, K. Li, and L. Fei-Fei. ImageNet: A Large-Scale Hierarchical Image Database. In CVPRO9, 2009.

[Frome et al., 2013] Andrea Frome, Greg S Corrado, Jon Shlens, Samy Bengio, Jeff Dean, Tomas Mikolov, et al. Devise: A deep visual-semantic embedding model. In $A d$ vances in Neural Information Processing Systems, pages 2121-2129, 2013.

[Girshick, 2015] Ross Girshick. Fast r-cnn. In Proceedings of the IEEE International Conference on Computer Vision, pages 1440-1448, 2015.

[Gong et al., 2013] Dihong Gong, Zhifeng Li, Dahua Lin, Jianzhuang Liu, and Xiaoou Tang. Hidden factor analysis for age invariant face recognition. In The IEEE International Conference on Computer Vision (ICCV), December 2013.

[Gong et al., 2015] Dihong Gong, Zhifeng Li, Dacheng Tao, Jianzhuang Liu, and Xuelong Li. A maximum entropy feature descriptor for age invariant face recognition. In The IEEE Conference on Computer Vision and Pattern Recognition (CVPR), June 2015.

[Guthrie et al., 2006] David Guthrie, Ben Allison, Wei Liu, Louise Guthrie, and Yorick Wilks. A closer look at skip-gram modelling. In Proceedings of the 5th international Conference on Language Resources and Evaluation (LREC-2006), pages 1-4, 2006.

[Hodosh et al., 2013] Micah Hodosh, Peter Young, and Julia Hockenmaier. Framing image description as a ranking task: Data, models and evaluation metrics. Journal of Artificial Intelligence Research, pages 853-899, 2013.

[Kiros et al., 2014] Ryan Kiros, Ruslan Salakhutdinov, and Richard S Zemel. Unifying visual-semantic embeddings with multimodal neural language models. arXiv preprint arXiv:1411.2539, 2014.

[Lazaridou et al., 2015] Angeliki Lazaridou, Nghia The Pham, and Marco Baroni. Combining language and vision with a multimodal skip-gram model. arXiv preprint arXiv:1501.02598, 2015.

[Mikolov et al., 2013] Tomas Mikolov, Ilya Sutskever, Kai Chen, Greg S Corrado, and Jeff Dean. Distributed representations of words and phrases and their compositionality. In Advances in neural information processing systems, pages 3111-3119, 2013.

[Norouzi et al., 2013] Mohammad Norouzi, Tomas Mikolov, Samy Bengio, Yoram Singer, Jonathon Shlens, Andrea
Frome, Greg S Corrado, and Jeffrey Dean. Zero-shot learning by convex combination of semantic embeddings. arXiv preprint arXiv:1312.5650, 2013.

[Perona, 2010] Pietro Perona. Vision of a visipedia. Proceedings of the IEEE, 98(8):1526-1534, 2010.

[Plummer et al., 2015] Bryan A Plummer, Liwei Wang, Chris M Cervantes, Juan C Caicedo, Julia Hockenmaier, and Svetlana Lazebnik. Flickr30k entities: Collecting region-to-phrase correspondences for richer image-tosentence models. In Proceedings of the IEEE International Conference on Computer Vision, pages 2641-2649, 2015.

[Schmid, 1995] Helmut Schmid. Treetagger- a language independent part-of-speech tagger. Institut für Maschinelle Sprachverarbeitung, Universität Stuttgart, 43:28, 1995.

[Simonyan and Zisserman, 2014] Karen Simonyan and Andrew Zisserman. Very deep convolutional networks for large-scale image recognition. arXiv preprint arXiv:1409.1556, 2014.

[Smith et al., 2013] Jason R Smith, Herve Saint-Amand, Magdalena Plamada, Philipp Koehn, Chris CallisonBurch, and Adam Lopez. Dirt cheap web-scale parallel text from the common crawl. In $A C L$ (1), pages 13741383, 2013.

[Szegedy et al., 2015] Christian Szegedy, Wei Liu, Yangqing Jia, Pierre Sermanet, Scott Reed, Dragomir Anguelov, Dumitru Erhan, Vincent Vanhoucke, and Andrew Rabinovich. Going deeper with convolutions. In Proceedings of the IEEE Conference on Computer Vision and Pattern Recognition, pages 1-9, 2015.

[Vijayanarasimhan and Grauman, 2014] Sudheendra Vijayanarasimhan and Kristen Grauman. Large-scale live active learning: Training object detectors with crawled data and crowds. International Journal of Computer Vision, 108(1-2):97-114, 2014.

[Zhu et al., 2015] Yuke Zhu, Ce Zhang, Christopher Ré, and Li Fei-Fei. Building a large-scale multimodal knowledge base for visual question answering. arXiv:1507.05670, 2015. 